\title{
ANATOMIA DO CARVÃO DE ESPÉCIES FLORESTAIS
}

\author{
Graciela Inés Bolzon de Muñiz ${ }^{1}$, Silvana Nisgoski1 ${ }^{1}$, Felipe Zatt Shardosin², Ramiro Faria França²
}

(recebido: 28 de outubro de 2010; aceito: 30 de março de 2012)

RESUMO: O carvão vegetal mantém a estrutura anatômica da madeira que o originou e pode permitir sua identificação botânica, a qual depende das características da espécie, o tamanho dos fragmentos do carvão e o estado de preservação. A caracterização anatômica do carvão de dez espécies florestais foi efetuada com o objetivo de auxiliar na identificação e controle de carvão ilegal. A diferenciação entre angiospermas e gimnospermas é bem evidente no lenho carbonizado. O diâmetro dos poros foi estatisticamente diferente entre a madeira e carvão na Vatairea guianensis, Mezilaurus itauba, Calophyllum brasiliense e Qualea cf. acuminata, e a frequência dos poros na Vatairea guianensis, Manilkara huberi, Qualea cf. acuminata e Simarouba amara. A estrutura anatômica da madeira, de maneira geral, se mantém durante o processo de carbonização utilizando-se temperatura de $450^{\circ} \mathrm{C}$, podendo-se identificar o material com base nas células componentes.

Palavras-chave: Lenho carbonizado, gimnospermas, angiospermas.

\section{CHARCOAL ANATOMY OF FOREST SPECIES}

ABSTRACT: Vegetal charcoal retains the anatomical structure of the wood and may permit its botanical identification, which depends on species characteristics, the charcoal fragments size and preservation state. Anatomical characterization of ten forest species charcoal was done envisaging the identification and control of illegal charcoal. Differences between gymnosperms and angiosperms are evident in carbonized wood. Vessel diameter was statistically different between wood and charcoal in Vatairea guianensis, Mezilaurus itauba, Calophyllum brasiliense e Qualea cf. acuminata, and vessel frequency in Vatairea guianensis, Manilkara huberi, Qualea cf. acuminata e Simarouba amara. The anatomical structure from wood, in general aspects, is constant during carbonization process using temperature of $450^{\circ} \mathrm{C}$, being possible to identify the material by using its cellular components.

Key words: Carbonized wood, gymnosperms, angiosperms.

\section{INTRODUÇÃO}

O carvão, produto final da carbonização ou o resultado de uma combustão incompleta mantém a estrutura anatômica da madeira que o originou e pode permitir sua identificação botânica, a qual depende das características anatômicas da espécie, o tamanho dos fragmentos do carvão e o estado de preservação (MARGUERIE; HUNOT, 2007).

A identificação de espécies a partir de madeira carbonizada é antiga, mas o processo era lento. Com o desenvolvimento de técnicas e equipamentos de microscopia a análise da estrutura anatômica do carvão tornou-se mais rápida, sendo também importante na antracologia, que é o estudo e interpretação de restos de lenho carbonizados, recolhidos em contexto arqueológico que, mediante amostragem e classificação, fornece informações importantes sobre paleoecologia e paleoetnologia (VERNET, 1987).

Amostras de carvão são especialmente valiosas na reconstrução histórica de sítios arqueológicos e para a interpretação de climas passados. Andreacci et al. (2009), estudando uma fogueira paleoíndia do Brasil Central, concluíram que três espécies puderam ser identificadas mediante a boa conservação da estrutura anatômica dos carvões. Estudos da anatomia do carvão nas análises paleobotânicas têm sido freqüentes, englobando, por exemplo, a identificação da distribuição natural das espécies (LUDEMANN et al., 2004), formação de ecossistemas naturais na era do Terciário e Quaternário (FIGUEIRAL; MOSBRUGGER, 2000), identificação das espécies do Holoceno na Etiópia (GEBRU et al., 2009) e China (KAISER et al., 2009), variação na umidade do ambiente (MARCONETTO, 2010), entre outros. No Brasil, trabalhos nessa área são recentes e efetuados por poucos pesquisadores, sendo ainda escassas as informações publicadas, envolvendo trabalhos de caracterização anatômica do carvão efetuados por Gonçalves et al. (2008), Gonçalves e Scheel-Ybert (2003) e Scheel-Ybert et al. (2006).

\footnotetext{
${ }^{1}$ Engenheira Florestal, Professora Doutora em Engenharia Florestal - Universidade Federal do Paraná/UFPR - Departamento de Engenharia e Tecnologia Florestal - Av. Pref. Lothário Meissner, 900 - Campus III - Jardim Botânico - 80.210-170 - Curitiba, PR, Brasil - gbmunize@ufpr.br, nisgoski@ufpr.br

${ }^{2}$ Acadêmico de Engenharia Industrial Madeireira - Universidade Federal do Paraná/UFPR - Av. Pref. Lothário Meissner, 900 - Campus III - Jardim Botânico -80.210-170 - Curitiba - PR, Brasil - zatt@ufpr.br, ramirofaria@hotmail.com
}

Cerne, Lavras, v. 18, n. 3, p. 471-477, jul./set. 2012 
No Brasil, embora tenha ocorrido um aumento significativo do uso de madeira de reflorestamento para a produção de carvão, uma grande parte é proveniente de madeiras nativas e derrubadas ilegais. Por outro lado, a carbonização de maneira artesanal é o meio de vida de muitos brasileiros, sendo que utilizam as árvores em volta do local onde residem para a carbonização, incluindo exemplares remanescentes de diversas espécies nobres que ainda não atingiram diâmetro comercial. Em função dessa exploração predatória dos recursos florestais, medidas de proteção para coibir o uso de madeiras extraídas ilegalmente têm sido propostas, mas encontram dificuldades no que se refere à identificação correta das espécies carbonizadas. Assim sendo, neste trabalho, objetivou-se a caracterização anatômica do carvão de dez espécies florestais, para ajudar na identificação e fornecer subsídios e auxílio no controle de carvão ilegal.

\section{MATERIAL E MÉTODOS}

Foram estudadas as seguintes espécies: araucária (Araucaria angustifolia (Bert.) Kuntze), castanheira (Bertholletia excelsa Humb. \& Bonpl.), faveira bolacha (Vatairea guianensis Aubl.), imbuia (Ocotea porosa (Ness \& Mart.ex Ness) L.Barroso), itaúba (Mezilaurus itauba (Meins.)Taub. ex Mez), jacareúba (Calophyllum brasiliense Cambess.), maçaranduba (Manilkara huberi (Ducke) Chevalier), mandioqueira (Qualea cf. acuminata Spruce ex Warn.), marupá (Simarouba amara Aubl.) e pinus (Pinus taeda), cujas amostras foram provenientes da xiloteca do Laboratório de Anatomia da Madeira, da Universidade Federal do Paraná.
As amostras de madeira foram cortadas nas dimensões de $2 \times 2 \times 5 \mathrm{~cm}$, orientadas nos três planos anatômicos. Foram carbonizadas 10 amostras de cada espécie, com teor de umidade de $12 \pm 1 \%$ recobertas por papel alumínio em forno mufla, em regime de carbonização descrito na Tabela 1.

As ilustrações da madeira e carvão foram obtidas em Estereomicroscópico Discovery 12, da Zeiss. A medição dos elementos constituintes foi efetuada utilizando o software Axio Vision Rel. 4.7, no mesmo equipamento, seguindo as normas da International Association of Wood Anatomists - IAWA (1989), utilizadas para a descrição anatômica de madeiras. A variação das dimensões entre a madeira e o carvão produzido foi avaliada pelo teste de Tukey a $95 \%$ de probabilidade.

\section{RESULTADOS E DISCUSSÃO}

A diferenciação entre angiospermas e gimnospermas é bem evidente no carvão vegetal, sendo que a maioria das espécies carbonizadas manteve a estrutura anatômica característica de cada uma de maneira integral.

As características mensuradas nas angiospermas são mostradas na Tabela 2, onde pode-se observar a influência da espécie no comportamento dos elementos vasculares sendo que, em algumas espécies, os vasos contraíram de maneira significativa (Vatairea guianensis, Mezilaurus itauba e Qualea cf. acuminata), e em outras estouraram (Calophyllum brasiliense), aumentando de tamanho pela carbonização no processo anteriormente descrito. A variação significativa no diâmetro dos vasos e na frequência de poros $/ \mathrm{mm}^{2}$ também foi relatada por

Tabela 1 - Regime de carbonização.

Table 1 - Carbonization process.

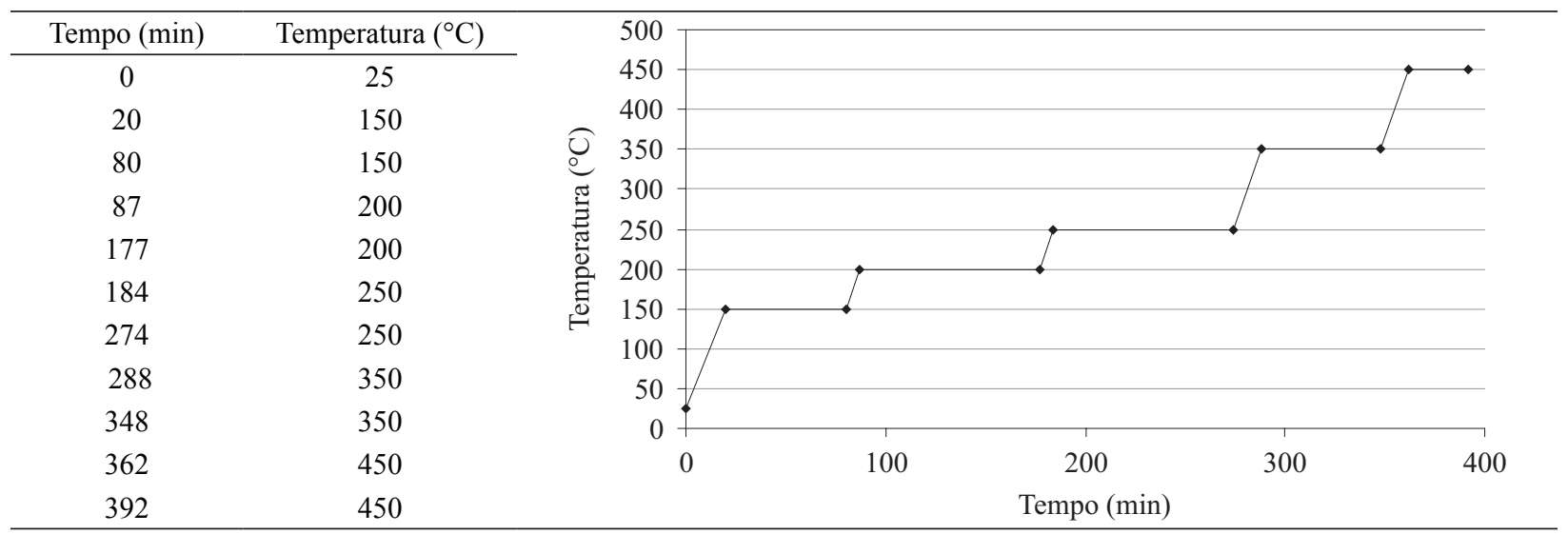

Cerne, Lavras, v. 18, n. 3, p. 471-477, jul./set. 2012 
Gonçalves (2010) para outras madeiras carbonizadas a $400{ }^{\circ} \mathrm{C}$ durante 40 minutos, indicando a influência da espécie na alteração.

Tabela 2 - Valores médios obtidos na caracterização anatômica quantitativa.

Table 2 - Mean values obtained in quantitative anatomic characterization.

\begin{tabular}{|c|c|c|c|c|}
\hline \multirow[t]{2}{*}{ Espécie } & \multicolumn{2}{|c|}{ Poros $/ \mathrm{mm}^{2}$} & \multicolumn{2}{|c|}{$\begin{array}{l}\text { Diâmetro dos } \\
\text { poros }(\mu \mathrm{m})\end{array}$} \\
\hline & Madeira & Carvão & Madeira & Carvão \\
\hline Bertholletia excelsa & $2,73^{\mathrm{a}}$ & $2,67^{\mathrm{a}}$ & $226,66^{\mathrm{a}}$ & $207,91^{\mathrm{a}}$ \\
\hline Vatairea guianensis & $2,00^{\mathrm{a}}$ & $3,13^{\mathrm{b}}$ & $142,08^{\mathrm{a}}$ & $96,01^{\mathrm{b}}$ \\
\hline Ocotea porosa & $9,90^{\mathrm{a}}$ & $9,37^{\mathrm{a}}$ & $102,26^{\mathrm{a}}$ & $104,28^{\mathrm{a}}$ \\
\hline Mezilaurus itauba & $8,63^{\mathrm{a}}$ & $8,50^{\mathrm{a}}$ & $160,25^{\mathrm{a}}$ & $135,02^{\mathrm{b}}$ \\
\hline Calophyllum brasiliense & $5,47^{\mathrm{a}}$ & $5,03^{\mathrm{a}}$ & $155,05^{\mathrm{a}}$ & $187,02^{\mathrm{b}}$ \\
\hline Manilkara huberi & $13,50^{\mathrm{a}}$ & $9,50^{\mathrm{b}}$ & $109,74^{\mathrm{a}}$ & $109,37^{\mathrm{a}}$ \\
\hline Qualea cf. acuminata & $3,13^{\mathrm{a}}$ & $5,47^{\mathrm{b}}$ & $182,98^{\mathrm{a}}$ & $148,12^{\mathrm{b}}$ \\
\hline Simarouba amara & $2,20^{\mathrm{a}}$ & $2,73^{\mathrm{b}}$ & $169,43^{\mathrm{a}}$ & $154,67^{\mathrm{a}}$ \\
\hline
\end{tabular}

*Médias seguidas pela mesma letra na linha, em cada característica, não diferem entre si pelo teste de Tukey a 95\% de probabilidade.
No lenho carbonizado da araucária (Figura 1b) e pinus (Figura 1d), as duas coníferas analisadas, é possível ver a distinção dos anéis de crescimento, com a diferente espessura da parede dos traqueoides, os raios finos e os canais resiníferos do pinus.

Entre as angiospermas, algumas espécies apresentaram rachaduras nos raios e nas linhas de parênquima, o que dificultou a visualização das características anatômicas componentes no plano transversal e a comparação com a madeira de origem.

A castanheira apresenta porosidade difusa, poros solitários em maioria, geminados e múltiplos radiais presentes, obstruídos por tilos; parênquima axial reticulado e raios finos no topo. A maçaranduba possui porosidade difusa, poros solitários pouco frequentes, múltiplos em cadeias radiais de 2 a 6 , obstruídos por tilos; parênquima axial formando um retículo irregular com os raios que são finos.

No lenho carbonizado da castanheira (Figura 2b), as rachaduras seguiram as linhas de parênquima, mas os vasos e raios permaneceram bem distintos, inclusive a obstrução dos vasos. Já, na maçaranduba (Figura 2d) as rachaduras no sentido dos raios prejudicaram por completo a identificação dos arranjos radiais dos poros e parênquima.

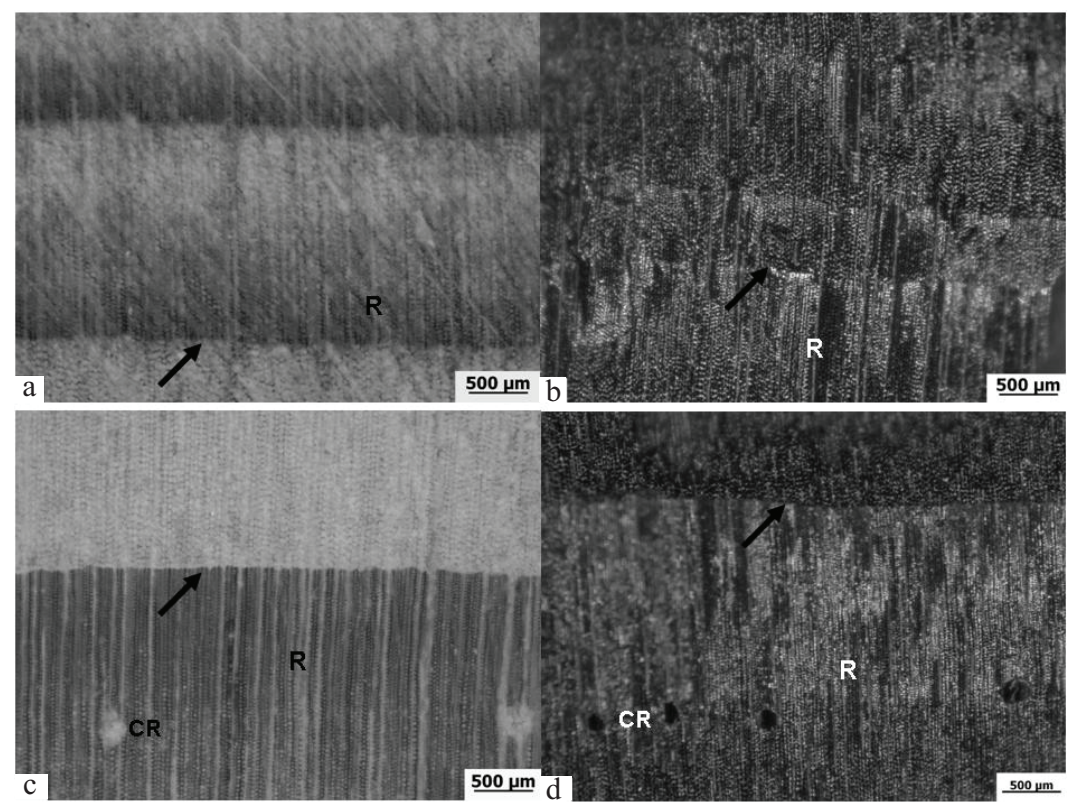

Figura 1 - Plano transversal da madeira e carvão de Araucaria angustifolia (a,b) e Pinus taeda (c,d). (R) Raio; (CR) Canal resinífero; seta: anel de crescimento.

Figure 1 - Transverse section of Araucaria angustifolia (a,b) and Pinus taeda (c,d) wood and charcoal. (R) Ray; (CR) Resin duct; arrow: growth ring.

Cerne, Lavras, v. 18, n. 3, p. 471-477, jul./set. 2012 

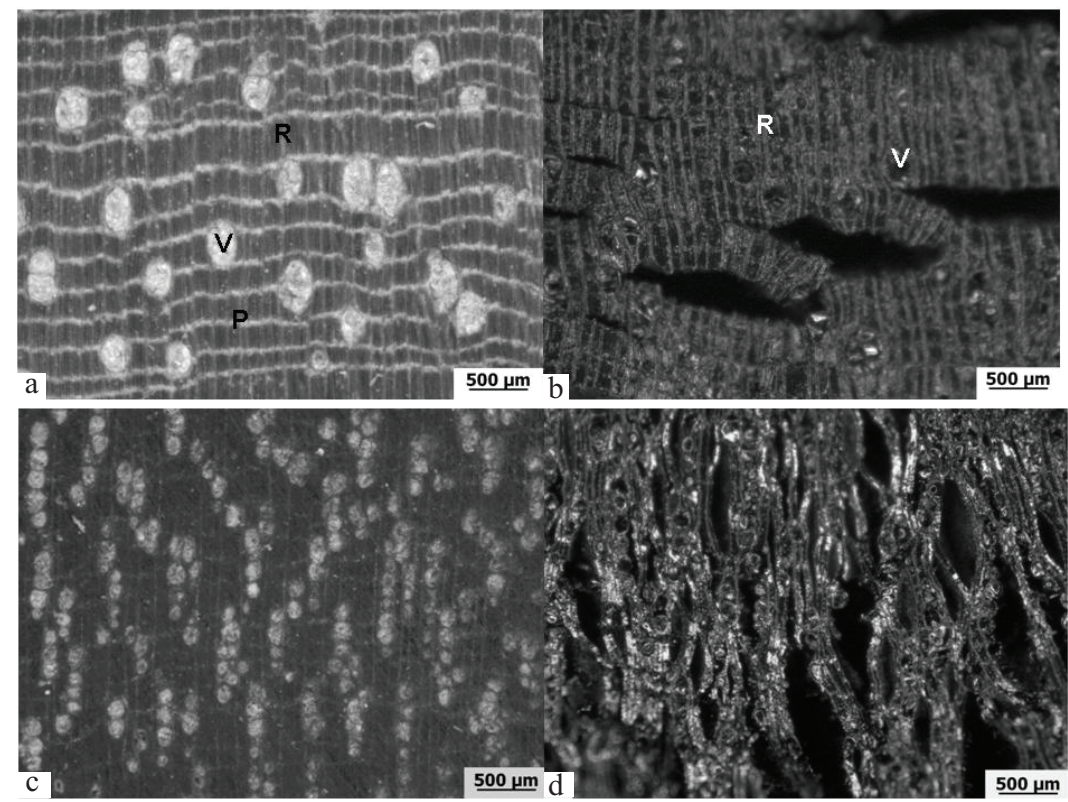

Figura 2 - Plano transversal da madeira e carvão de Bertholletia excelsa(a,b) e Manilkara huberi (c,d). (V) vaso; (P) parênquima; (R) raios.

Figure 2 - Transverse section of Bertholletia excelsa $(a, b)$ and Manilkara huberi $(c, d)$ wood and charcoal. $(V)$ vessel; $(P)$ parenchyma; ( $R$ ) rays.

A imbuia e a itaúba apresentam porosidade difusa, poros solitários em maioria, geminados e múltiplos presentes, obstruídos por óleo resina e tilos. Células oleíferas junto aos vasos e parênquima. Parênquima axial escasso e vasicêntrico. Camadas de crescimento distintas pelo espessamento da parede das fibras. Raios finos.

As amostras carbonizadas de imbuia (Figura 3b) e itaúba (Figura 3d) mantiveram bem visíveis os arranjos dos vasos e a distinção dos raios, com rachaduras em alguns deles, mas sendo possível a diferenciação dessas duas espécies da família Lauraceae, muito utilizadas para carbonização ilegal. Todas as amostras carbonizadas para as espécies apresentaram o mesmo tipo de rachadura.

A faveira bolacha e a mandioqueira apresentam porosidade difusa, poros solitários, geminados e múltiplos até três presentes, obstruídos por óleo resina densa e compacta. Parênquima axial paratraqueal aliforme losangular e confluente em trechos curtos. Raios finos e bem destacados. Fibras de paredes espessas.

No lenho carbonizado das amostras de faveira bolacha (Figura 4b) e mandioqueira (Figura 4d), observase que os vasos ficaram desobstruídos, evidenciando-se o agrupamento múltiplo, também sendo bem evidentes os raios e menos perceptível, nesse aumento, o parênquima axial.
A jacareúba apresenta porosidade difusa, poros solitários em maioria, geminados e múltiplos presentes, obstruídos por tilos e óleo resina. Parênquima axial em linhas curtas e longas. Raios finos e pouco distintos. O marupá possui porosidade difusa, poros solitários, geminados e múltiplos radiais, obstruídos presentes. $\mathrm{O}$ parênquima axial é paratraqueal aliforme linear e confluente em trechos curtos. Raios médios e bem destacados.

No lenho carbonizado da jacareúba (Figura 5b) observa-se, nitidamente, o arranjo dos poros e as finas linhas de parênquima axial. No caso do marupá (Figura 5d), como a madeira é clara, as finas linhas de parênquima aliforme não são visíveis nesse aumento, ao contrário dos raios e porosidade.

Analisando-se o comportamento geral das espécies, as rachaduras foram constantes para as espécies e são causadas pelo aquecimento e decomposição da superfície, mais rápido que o interior e, também, fortemente influenciadas pela orientação da grã. Essas variações também foram observadas por Byrne e Nagle (1997).

A análise da estrutura da madeira e carvão, em um estereomicroscópio de luz refletida utilizando-se apenas

Cerne, Lavras, v. 18, n. 3, p. 471-477, jul./set. 2012 

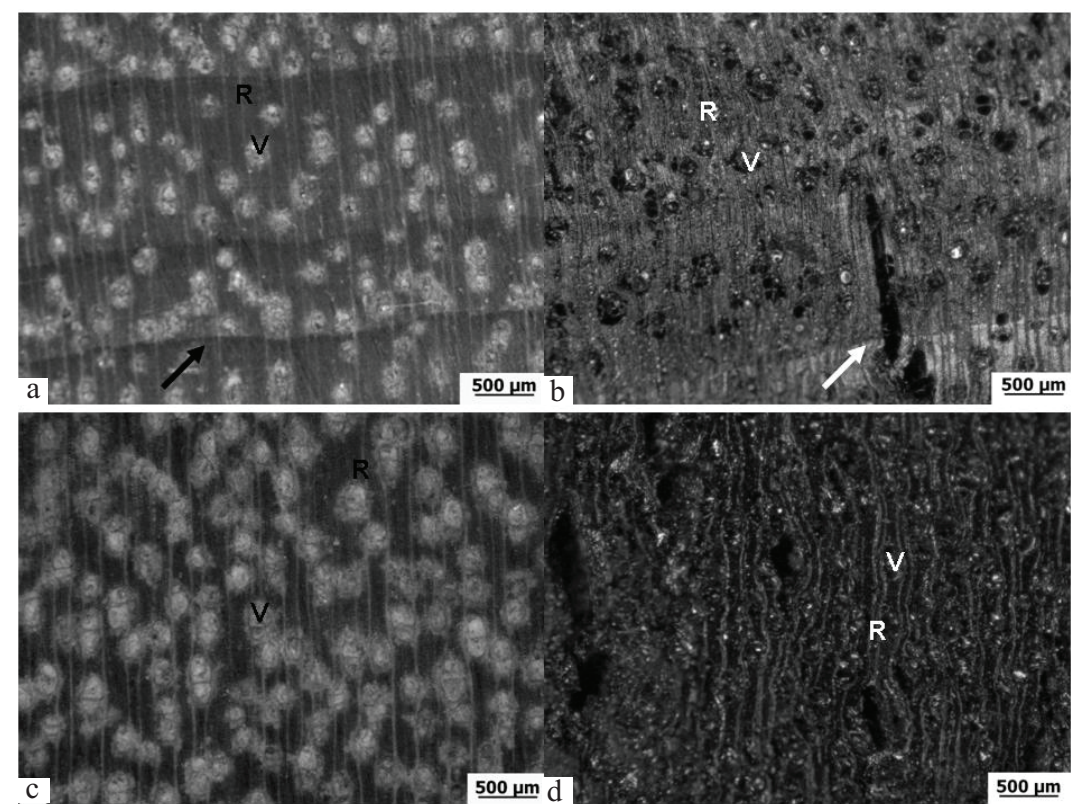

Figura 3 - Plano transversal da madeira e carvão de Ocotea porosa (a,b) e Mezilaurus itauba (c,d). (V) vaso; (R) raios; seta: anel de crescimento.

Figure 3 - Transverse section of Ocotea porosa $(a, b)$ and Mezilaurus itauba $(c, d)$ wood and charcoal. (V) vessel; (R) rays; arrow: growth ring.
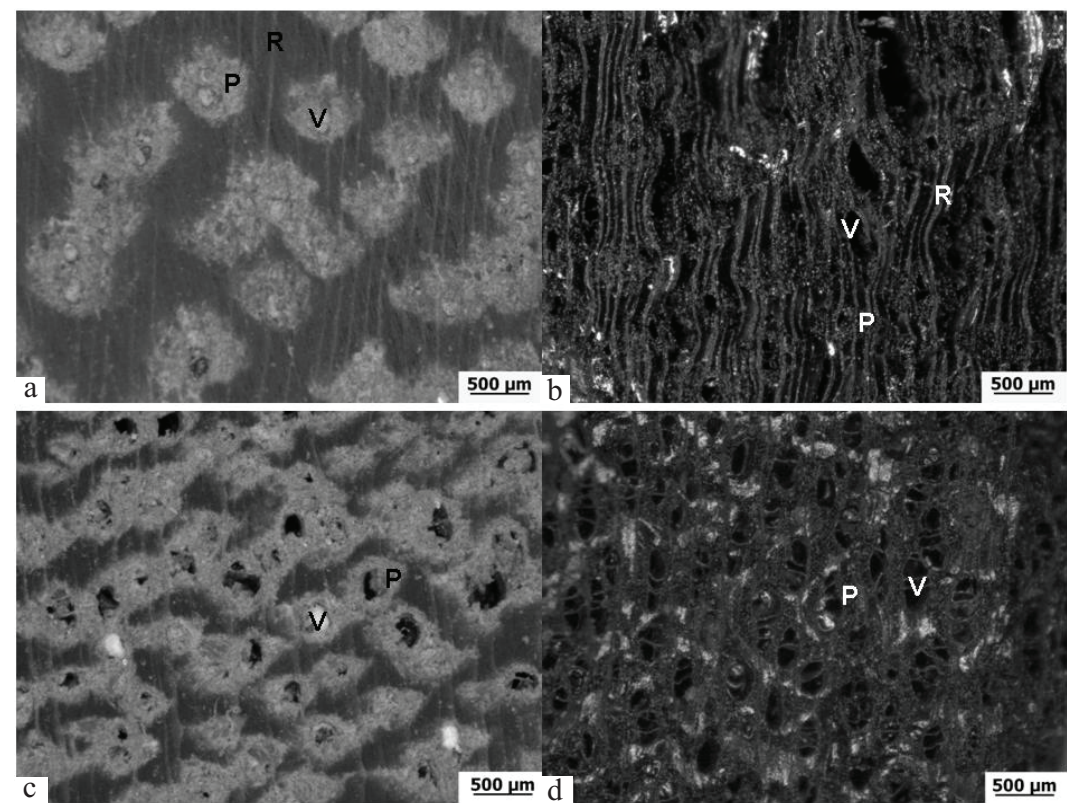

Figura 4 - Plano transversal da madeira e carvão de Vatairea guianensis (a,b) e Qualea cf. acuminata (c,d). (V) vaso; (P) parênquima; (R) raios.

Figure 4 - Transverse section of Vatairea guianensis $(a, b)$ and Qualea cf. acuminata $(c, d)$ wood and charcoal. (V) vessel; (P) parenchyma; $(R)$ rays.

Cerne, Lavras, v. 18, n. 3, p. 471-477, jul./set. 2012 

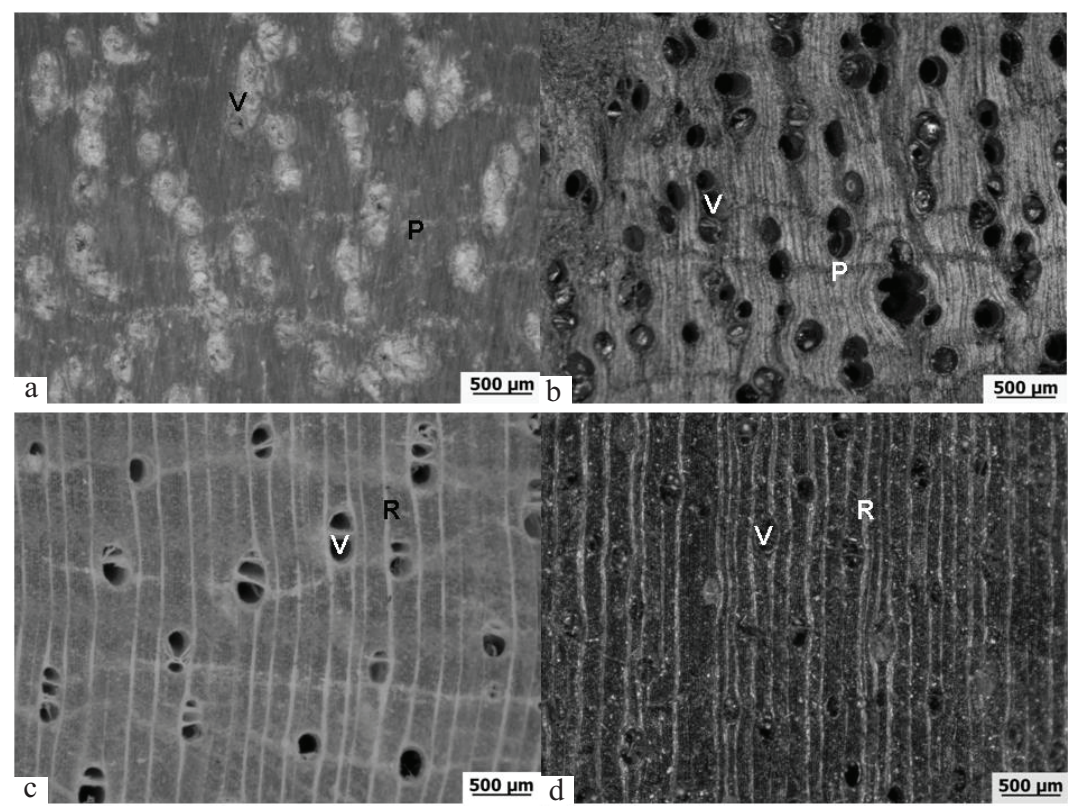

Figura 5 - Plano transversal da madeira e carvão de Calophyllum brasiliense (a,b) e Simarouba amara (c,d). (V) vaso; (P) parênquima; (R) raios.

Figure 5 - Transverse section of Calophyllum brasiliense $(a, b)$ and Simarouba amara $(c, d)$ wood and charcoal. $(V)$ vessel; $(P)$ parenchyma; $(R)$ rays.

aumentos de 10 e $25 \mathrm{x}$ mostrou que o aspecto anatômico geral de distribuição e tipos de células é bem evidente, sendo possível a identificação de material carbonizado com base na estrutura da madeira. Em alguns casos não é possível a identificação específica em campo, mas pelas características gerais observadas, um direcionamento de grupos pode ser efetuado, com a exclusão de algumas espécies potenciais.

\section{CONCLUSÃO}

A estrutura anatômica da madeira se mantém durante o processo de carbonização utilizado, com temperatura final de $450^{\circ} \mathrm{C}$, podendo-se identificar o material com base nas células componentes. A influência de diferentes taxas de aquecimento e umidade do material nas alterações estruturais devem ser estudadas. É muito importante, para a correta identificação, a nível de espécie, a comparação com uma coleção de referência.

No controle do carvão ilegal, a possibilidade de visualização de um grupo de características principais específicas em cada madeira é muito importante pela praticidade e rapidez de ação local.

Cerne, Lavras, v. 18, n. 3, p. 471-477, jul./set. 2012

\section{REFERÊNCIAS}

ANDREACCI, F. et al. Análise antracológica de uma fogueira paleoíndia do Brasil Central: um olhar sobre o uso pré-histórico de recursos florestais. In: ENCONTRO INTERNACIONAL DE PRODUÇÃO CIENTÍFICA CESUMAR, 6., 2009, Maringá. Anais... Maringá: CESUMAR, 2009. 1 CD-ROM.

BYRNE, C. E.; NAGLE, D. C. Carbonization of wood for advanced materials applications. Carbon, Elmsford, v. 35, n. 2, p. 259-266, 1997.

FIGUEIRAL, I.; MOSBRUGGER, V. A review of charcoal analysis as a tool for assessing Quaternary and Tertiary environments: achievements and limits. Palaeogeography, Palaeoclimatology, Palaeoecology, Amsterdam, n. 164, p. 397-407, 2000.

GEBRU, T. et al. Holocene palaeovegetation of the Tigray Plateau in northern Ethiopia from charcoal and stable organic carbon isotopic analyses of gully sediments. Palaeogeography, Palaeoclimatology, Palaeoecology, Amsterdam, n. 282, p. 67-80, 2009. 
GONÇALVES, T. A. P. Anatomia do lenho e carvão de espécies arbóreas em áreas de cerrado do Estado de São Paulo. 2010. 106 f. Dissertação (Mestrado em Ciência Florestal) - Universidade Estadual Paulista, Botucatu, 2010.

GONÇALVES, T. A. P. et al. Caracterização anatômica do lenho carbonizado de espécies comuns do cerrado: subsídio a estudos antracológicos e conservacionistas. In: SIMPÓSIO NACIONAL CERRADO, 9.; SIMPÓSIO INTERNACIONAL SAVANAS TROPICAIS, 2., 2008, Brasília. Anais... Brasília: ParlaMundi, 2008. 1 CD-ROM.

GONÇALVES, T. A. P.; SCHEEL-YBERT, R. Anatomia do lenho de espécies de Anacardiaceae e Annonaceae em amostras carbonizadas. In: JORNADA FLUMINENSE DE BOTÂNICA, 12.; REUNIÃO CIENTÍFICA DA SOCIEDADE BRASILEIRA DE BOTÂNICA, 44., 2003, Cabo Frio.

Resumos... Cabo Frio: SBB, 2003. p. 28.

\section{INTERNATIONAL ASSOCIATION OF WOOD}

ANATOMISTS. List of microscopic features for hardwood identification. IAWA Bulletin, Leiden, v. 10, n. 3, p. 219-332, 1989.

KAISER, K. et al. Charcoal and fossil wood from palaeosols, sediments and artificial structures indicating Late Holocene woodland decline in southern Tibet, China. Quaternary Science Reviews, Oxford, v. 28, p. 1539-1554, 2009.
LUDEMANN, T. et al. Spatial patterns of past wood exploitation, natural wood supply and growth conditions: indications of natural tree species distribution by anthracological studies of charcoal-burning remains.

European Journal of Forest Research, Georgetown, v. 123, p. 283-292, 2004.

MARCONETTO, M. B. Paleoenvironment and anthracology: determination of variations in humidity based on anatomical characters in archealogical plant charcoal (Ambato Valley, Catamarca, Argentina). Journal of Archaeological Science, New York, v. 37, p. 1186-1191, 2010 .

MARGUERIE, D.; HUNOT, J. Y. Charcoal analysis and dendrology: data from archaeological sites in north-western France. Journal of Archaeological Science, New York, v. 34, p. 1417-1433, 2007.

SCHEEL-YBERT, R. et al. Coleções de referência e banco de dados de estruturas vegetais: subsídios para estudos paleoecológicos e paleoetnobotânicos. Arquivos do Museu Nacional, Rio de Janeiro, v. 64, p. 255-266, 2006.

VERNET, J. L.; THIEBAULT, S. An approach to northwestern Mediterranean recent prehistoric vegetation and ecologic implications. Journal of Biogeography, Oxford, v. 14, p. 117127, 1987.

Cerne, Lavras, v. 18, n. 3, p. 471-477, jul./set. 2012 
